\title{
De l'Égypte à l'Inde, de Jean Cocteau (Maalesh) à Jean-Christophe Bailly (Phèdre en Inde)
}

\section{Françoise Simonet-Tenant}

\section{(2) OpenEdition}

\section{Journals}

\section{Édition électronique}

URL : http://journals.openedition.org/rief/975

DOI : $10.4000 /$ rief. 975

ISSN : 2240-7456

\section{Éditeur}

Seminario di filologia francese

\section{Référence électronique}

Françoise Simonet-Tenant, « De l'Égypte à l'Inde, de Jean Cocteau (Maalesh) à Jean-Christophe Bailly ( Phèdre en Inde) », Revue italienne d'études françaises [En ligne], 1 | 2011, mis en ligne le 15 décembre 2011, consulté le 20 avril 2019. URL : http://journals.openedition.org/rief/975 ; DOI : 10.4000/rief.975

Ce document a été généré automatiquement le 20 avril 2019.

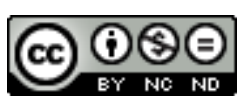

Les contenus de la RIEF sont mis à disposition selon les termes de la Licence Creative Commons Attribution - Pas d'Utilisation Commerciale - Pas de Modification 4.0 International. 


\title{
De l'Égypte à l'Inde, de Jean Cocteau ( Maalesh) à Jean-Christophe Bailly ( Phèdre en Inde)
}

\author{
Françoise Simonet-Tenant
}

1 De mars à mai 1949, Jean Cocteau part avec sa famille d'acteurs, sa famille des Parents terribles, pour une tournée théâtrale dans le bassin méditerranéen (Égypte, Liban, Turquie, Grèce). De cette tournée, il rapporte un Maalesh, Journal d'une tournée de théâtre (Gallimard, 1949). D'août 1989 à janvier 1990, Jean-Christophe Bailly séjourne à trois reprises en Inde pour créer Phèdre avec des acteurs indiens et en revient avec Phèdre en Inde (Plon, 1990). Cocteau et Bailly ne sont pas de simples voyageurs touristes mais des hommes de théâtre dont le journal de voyage est également un journal d'expérience professionnelle. Dans ce contexte, nous tenterons de dégager les fonctions du journal et la posture du diariste vis-à-vis de la publication; nous observerons également la manière dissemblable dont les deux diaristes appréhendent et traduisent l'expérience sensible de l'altérité.

2 Les journaux de voyage sont traditionnellement animés par un double mouvement, d'extraversion - les diaristes notent les choses vues - et d'intraversion - ils notent le retentissement du choc de l'ailleurs. Dans une belle définition paradoxale, Bailly affirme : «Voyager, c'est être immobile, c'est aller à la rencontre de l'immobilité dans ses formes locales, pour que tout claque au vent ensuite, à l'intérieur $»^{1}$. Quels rôles peut tenir le journal lors de l'expérience de l'étranger et de la possible étrangeté ? Bien évidemment, il est trace mémorielle : « il ne peut s'agir que de former une trace, si possible ressemblante, et marquée au coin, comme toute autre, de la présomption qu'il y a à en laisser : mais si je n'en avais rien écrit, il me semble que cette expérience m'échapperait, c'est aussi simple » (PhI, p. 180). Sans doute le journal est-il plus qu'un simple support mémoriel. Il est aussi une indispensable boussole, un carnet de jalons pour l'occidental dépaysé. C'est ainsi que Cocteau confie à son journal ses doutes, les moments de vacillement où le voyageur ne 
sait plus très bien ce qu'il est venu faire dans un lieu étranger, les moments où les motivations du voyage se brouillent :

Pourquoi trimbaler d'immenses décors et vingt-deux artistes dans des villes qui dorment debout et qu'on réveille à l'improviste avec des phrases qui sortent d'un autre monde que le leur, et pourquoi l'Égypte et la Turquie plutôt que l'Espagne, le Brésil, la Suède ou la Norvège, et pourquoi se donner tant de mal et aller si loin, grand Dieu, pour voir en fin de compte des personnes inconnues claquer leurs pattes de devant ??

3 Pour s'approprier une réalité dépaysante et déconcertante, la comparaison reste un outil efficace. Le bagage de rhétorique est indispensable au voyageur en manque de repères. Bailly multiplie les comparaisons pour donner à la réalité inconnue le visage familier d'une réalité reconnue :

L'atmosphère de Hamydia Road me rappelle celle des avenues de Mombasa, avec moins de lumière mais davantage de monde. (PhI, p. 34-35)

Le Jantar Mantar, l'observatoire de Jaïpur, est beau, plus que beau même. L'architecture la plus réussie ne fait souvent qu'impressionner. Rarement, elle libère. Or, c'est le cas ici, comme dans les jardins de Kyoto, comme parfois en Italie. (PhI, p. 46-47)

4 Au-delà des comparaisons, Bailly se caparaçonne de lectures, pour affronter le choc de l'inconnu. Ce sont d'abord les lectures préparatoires conçues comme ressource nécessaire pour décrypter l'altérité: ainsi il lit L'Inde brisée de Naipaul (PhI, p.16), les Souvenirs d'enfance de Tagore (PhI, p. 16), L'Odeur de l'Inde de Pasolini (PhI, p. 17), le Voyage au-delà des trois mers d'Athanase Nikitine (PhI, p. 20), L'Inde fondamentale de Louis Renou (PhI, p. 22), la Gita-Govinda et Le Danseur de cour de Lokenath Bhattacharya (PhI, p. 63), un article de Charles Malamoud sur l'« opposition, dans l'Inde ancienne, entre le vaste et resserré, [la] complétude entre le plein et le creux » (PhI, p. 64). Le diariste, voyageur et lecteur, est néanmoins conscient du pouvoir heuristique limité des lectures :

Vouloir connaître un peu mieux ou un peu moins mal la civilisation d'un pays où l'on se rend, ce mouvement est simple. Il se complique pourtant très vite. Une civilisation, c'est un monde et une forme du monde, des choses devant la vue et un regard sur ces choses, c'est une profondeur enfouie et un envol d'apparences. Aussi, entre ce qui est vu et ce qui regarde, comme entre ce qui s'est entreposé avec le temps et ce qui se contente d'apparaître, les livres émergent-ils comme les meilleurs intercesseurs. Mais ce sont aussitôt mille et une rubriques dans mille et un livres, ce sont des bibliographies qui à la fois ou selon les jours découragent ou émerveillent. Se teinter d'un peu de culture aussi bien n'est pas le but. Chaque livre exerce une trouée en un point précis, chaque livre, même s'il renvoie aux autres, est tout de même solitaire. Mais demeure, de quelque façon qu'on s'y prenne, le mystère d'un descellement entre ce que l'on apprend et ce que l'on éprouve sur place. (PhI, p. 60-61)

5 Les lectures préparatoires du voyage s'accompagnent de lectures symétriques quand le voyageur est sur le lieu de son voyage. C'est alors le temps de lire des grands textes, ceux qui concentreraient une manière de quintessence de l'esprit européen :

Hier soir j'ai commencé Anna Karénine. Livres qu'on lit très jeune ou, en n'y prenant pas garde, jamais. Tolstoï est si efficace lorsqu'on tient à s'isoler, à se couper du flux de ses propres impressions. (PhI, p. 35)

Mettant à profit les lointains séjours pour combler quelques trous trop voyants à mes yeux, j'ai emporté cette fois Les années d'apprentissage de Wilhelm Meister, que j'ai commencé à lire le soir, et ce soir avec plus d'entrain. Par-delà le mystère goethéen de l'alliance malgré tout supportable entre le sublime et le moralisant, entre ce qui est enlevé et ce qui est épais, et malgré cette odeur de soupe fumante qui n'est 
jamais trop loin du terrain des aventures et qui est « son pays du soir » à lui, le livre de Goethe remplit aisément la fonction qu'en l'emportant ici je lui assignais, et qui est d'incarner autant que possible et parfois jusqu'à l'écœurement l'Europe de la tradition. (PhI, p. 90-91)

Jean-Christophe Bailly observe l'Inde à travers le filtre des références culturelles accumulées qui sont les siennes :

L'immobilité du paysage et de l'eau sans reflets vraiment comme un prodige, l'expression «pas un souffle » devenue vraie, devenue ville, Bhopal tel l'autre côté du Rivage des Syrtes. (PhI, p. 88)

Trains de banlieue aux flancs lourds qui se vident et se remplissent, les cireurs de chaussures frappent à coups réguliers leurs étals pour attirer les clients, juste à la sortie des quais, devant la gare, une mendiante, entièrement nue, avait l'expression de la folle de Géricault. (PhI, p. 102)

7 À cette posture de lettré, Cocteau oppose un prétendu regard vierge :

L'Acropole. J'y retourne. Quelle chance de ne pas être un intellectuel! Quelle chance de ne pas être un homme de parti! Quelle chance d'être un absurde sur cette étrange plate-forme, sur cet aérodrome, sur ce train d'atterrissage des dieux. Bien sûr, je sais certaines choses, on m'a dit certaines choses, j'ai lu Renan et Maurras. Mais, au soleil, je suis du vide en face d'un vide peu encombré de quelques objets de travers. (M, p. 217)

8 Quelles que soient les stratégies déployées par l'un et l'autre pour tenter de saisir une réalité et une expérience qui se dérobent aux formes discursives, les journaux de Cocteau et de Bailly pourraient s'intituler Choses vues. L'on remarque, à la lecture de ces deux journaux écrits à quelque quarante années de distance, l'écrasante prépondérance du sens visuel dans l'appréhension de l'étranger. Bailly et Cocteau s'en tiennent aux seules ressources du langage pour rendre compte de la réalité foisonnante. On n'est pas encore à l'heure des blogs de voyage du XXI siècle où le texte occupe souvent une place modeste dans la collecte archivistique des impressions de l'étranger, qui donne la part belle aux photographies, aux prises de son et même aux petits films. Cocteau, en esthète, décrit souvent les lieux traversés comme un décor de théâtre. Bailly dans un journal de voyage ultérieur déclare que, pour lui, « le carnet est devenu et est resté l'outil du voyage, en lieu et place de l'appareil photo $"^{3}$. Les énumérations par accumulation sont présentes dans les deux journaux. Les deux diaristes, soucieux de dire les choses vues, déplient dans de longues énumérations l'immédiateté d'un regard saturé de visions, ce que Bailly appelle «la violence pure de l'impression, poing humide d'images collées sur la rétine » (PhI, p. 24) :

Acropole. [...] Le chaos, couleur de pierre ponce, les massifs d'héliotrope pétrifiés, les membres épars du grand corps écartelé debout au milieu des éclaboussures de sang des coquelicots, les cages à dieux, grandes et petites, ouvertes, suspendues en l'air dans tous les sens. (M, p. 206)

(Beignets en train de frire devant un tas de pneus, vaches qui somnolent dans le tumulte, affalées sur le terre-plein central d'une route à deux voies, pieds nus et klaxons, bracelets et saris, envols des couleurs des femmes et incroyable grisaille des vêtements des hommes, tristes chemisettes et tristes pantalons, gens qui poussent et gens qui stagnent, charges inédites et mains vides, regards béants, pieds qui traînent, scooters et vélos qui s'infiltrent toujours, petits fanions, guirlandes de feuilles séchées suspendues à des cabanes, feux à même le sol, tenanciers accroupis, singes, corbeaux, porcs qui ramassent et trient la fange - tout ce que l'on se surprend à aimer et qui, selon les jours, ressemble à un désastre ou au contraire à une effusion lyrique de la vie). (PhI, p. 163-164) 
Bailly, en intellectuel soucieux de ne pas succomber à l'immédiateté de ses impressions, multiplie les précautions métadiscursives; il argue de la vérité inévitable de certains clichés et de l'impossibilité parfois d'échapper à l'emprise du pittoresque :

Nous finissons par prendre le train de nuit à la gare. La légende est là, vraie : les guichets fermés, les informations contradictoires, l'agitation de la foule sur les quais comme s'il y avait exode, et surtout, dans le hall, les corps couchés à même le sol dans des chiffons blancs qui ont l'air de linceuls, l'antichambre des limbes qu'est toute gare indienne dès le jour tombé. On a beau le savoir, on a beau en prendre son parti et, comme tout le monde, enjamber les corps endormis, l'image traverse le cliché et agrippe comme une main. (PhI, p. 44)

Le journal de voyage est un type de journal particulier : s'ouvrant avec le début du voyage et se fermant avec sa fin, il possède une unité intrinsèque qui en favorise l'éventuelle publication. De mars à mai 1949, Cocteau part, avec vingt-deux comédiens dont Jean Marais, Yvonne de Bray, Gabrielle Dorziat, Gaby Sylvia, pour une tournée théâtrale qui les conduit successivement en Égypte, au Liban, en Turquie et en Grèce. Le programme est éclectique : les comédiens représentent des pièces de Racine (Britannicus), de Feydeau ( Léonie est en avance), de Sartre (Huis clos), d'Anouilh (Léocadia) et de Cocteau lui-même (Les Parents terribles, La Machine infernale, Les Monstres sacrés). C'est sur l'Égypte que les notes du journal sont les plus nombreuses et les plus fournies. Le voyage est itinérant, les lieux de représentation divers, et les trajets en avion suscitent quelques commentaires chez Cocteau à une époque où le voyage aéronautique a encore un parfum d'aventure : «J'ai cessé d'écrire parce que je me sentais assez malade. Je n'étais pas le seul. Le modèle d'appareil où nous sommes ne possède aucune bouche d'aération. Il n'y circule pas un pouce d'air. [...] J'ai quitté l'avion, à Héliopolis, ridiculement pâle et mort de fatigue » (M, p. 32-33). Le genre de voyage entrepris par Jean-Christophe Bailly est sensiblement différent. Trois séjours successifs en Inde, d'août 1989 à janvier 1990, fournissent les matériaux de son journal (5-16 août, 4 novembre-25 novembre, 10 décembre-16 janvier). Georges Lavaudant et Jean-Christophe Bailly doivent aider à la création d'une pièce en Inde, issue du répertoire français, interprétée en hindi par des comédiens d'une troupe de Bhopal. La pièce de Phèdre est choisie. Dès lors, ce qui constitue le fil rouge du journal est le « devenir indien » (PhI, p. 65) de Phèdre, comme l'assure Bailly. Dès la note préliminaire qui précède le journal, Bailly écrit : «Approcher la vibration propre à une terre, tâcher d'en restituer la résonance et les effets sur un texte transporté là-bas depuis notre tradition, tels sont donc les buts de ces carnets » (PhI, p. 12). Greffer Phèdre sur la terre et sur la langue indiennes est une expérience singulière et imprévisible. Le journal, écriture à l'aveuglette, ignorante de son lendemain, est donc particulièrement bien approprié à la saisie d'une telle expérience: «Dans le cas précis de cette Phèdre étrangée, toute idée préconçue eût été fausse. Impossible de venir avec un plan de route quand c'est la route elle-même qui naît sur place" (PhI, p.120). La route naîtra sur place jusqu'à la représentation : «La première a eu lieu. Les cérémonies d'adieu, très émouvantes, sont terminées. [...] Au théâtre tout commence quand tout est fini » (PhI, p. 152). Encore quelques pages, et le journal de cette "Phèdre étrangée " prendra fin. Journal d'une expérience théâtrale ou journal de tournée, dans les deux cas, le journal se clôt de manière nette. 
11 Les deux diaristes avaient-ils à l'esprit, lors de sa tenue, la publication du journal de voyage? Les journaux tels qu'ils sont publiés ont-ils fait l'objet de réécritures? Certes, les deux journaux obéissent à un protocole de modestie qui pourrait être l'autre visage de l'authenticité. Cocteau affirme: «Un journal est un journal sous peine de perdre son style. Je dois m'y contredire, apprendre en route, laisser mes erreurs où elles se trouvent, ne pas avoir honte de m'y montrer le perpétuel élève que nous sommes» ( $M$, p. 104). Quant à Bailly, il avertit le lecteur: "Je ne puis trier les copeaux, l'ivraie est ici abondante, mais c'est la règle du jeu, et le journal de voyage est un genre aussi exigeant qu'un autre. Le but n'est d'ailleurs jamais celui de laisser quelques "bonnes pages", il ne peut s'agir que de former une trace, si possible ressemblante» (PhI, p. 180). Néanmoins Bailly a précisé dans sa note liminaire :

Écrits au jour le jour et presque quotidiennement, du moins quand j'étais sur place, ils commencent quelques semaines avant mon premier départ et s'achèvent à Delhi, quelques heures avant mon retour définitif en France. Je les ai tapés régulièrement à mes retours, en triant le moins possible, même s'il va de soi que certaines remarques de pure humeur ou dont la lecture révélait le caractère redondant devaient disparaître. (PhI, p. 12)

Le diariste, devenu son propre éditeur, ne se serait ainsi permis qu'une discrète autocensure esthétique. Les "quelques bonnes pages» que le diariste affecte de dédaigner ne sont cependant pas tout à fait absentes du volume. Bailly et Cocteau sacrifient tous deux à la tradition du morceau de bravoure descriptif qui dilate certaines entrées du journal : il en va ainsi chez Bailly de la description d'Elephanta, petite île de la baie de Bombay (PhI, p. 104-108) ou de l'évocation de Louxor et Karnak par Cocteau ( $M$, p. 99-122). On soupçonne que de tels passages - néanmoins rares chez Bailly - ont pu être composés après coup à partir de notes rapides : la narration n'échappe pas entièrement à l'influence du style du reportage et il n'est pas simple alors de faire le partage entre diarisme et journalisme. La situation du texte dans cet entre-deux n'est pas nouvelle: depuis 1850, la presse, consciente de l'emballement du public pour le récit de voyage, a ouvert ses colonnes à des récits de voyage publiés en chroniques et à des reportages présentés comme des lettres ou des journaux de voyage. Le glissement du journal personnel vers la chronique journalistique n'étonne pas chez Cocteau dont ce fut, un temps, le métier. Chroniqueur dans la presse des années 1930, Cocteau a entrepris avec son ami Marcel Khill un nouveau tour du monde en 80 jours qui a donné lieu à des chroniques, publiées par Paris-Soir ; les chroniques furent recueillies en un seul volume publié chez Gallimard en 1936, intitulé Mon premier voyage et dédié à André Gide, figure tutélaire du diarisme voyageur. La conscience d'un public à étonner, intéresser et séduire est toujours présente à l'esprit de Cocteau qui, après avoir décrit longuement une représentation de Britannicus à Istanbul, ajoute : «Je me suis amusé à écrire le reportage d'une de nos représentations - qu'on m'en excuse - mais ce livre s'adresse aux quelques fous qui se passionnent encore pour le travail des planches » ( $M$, p. 169). Les adresses au lecteur ponctuent le journal de Cocteau et suggèrent qu'à l'horizon de l'écriture du diariste voyageur ou du journaliste confidentiel se dessine la perspective de la publication. Chez Bailly, la présence explicite du lecteur est rare, réduite à une seule occurrence, presque parodique, à l'avant-dernière page du journal : «J'ai vu l'Inde sans doute, je l'ai aperçue, elle est venue se déposer d'elle-même dans les recoins de ces pages à qui le goût que j'ai des journaux de voyage procure une manière de sauf-conduit, et vers toi, lecteur, comme on disait autrefois » (PhI, p. 179). Un autre journal de voyage, très 
récemment publié par Bailly et intitulé Dans l'étendu, explicite son goût pour les journaux de voyage et revient sur les conditions de genèse de Phèdre en Inde:

Une seule fois, dans les notes que j'ai tenues en Inde au moment où je travaillais làbas avec Georges Lavaudant à la mise en scène de Phèdre, j'ai pu tenir constamment ou presque un vrai journal de bord circonstancié et à peu près écrit: mais d'une part il y avait là un axe bien précis et, aussi, une condition plutôt sédentaire qui me permettaient d'écrire de la sorte et, d'autre part, c'est tout à fait consciemment que j'écrivais ces notes en vue d'une publication: Alain Veinstein m'ayant invité quelque temps avant mon départ pour l'Inde à publier quelque chose dans la Collection « Carnets » qu'il dirigeait alors chez Plon, c'est tout naturellement que je lui avais évoqué les notes que j'allais, je le savais, recueillir en Inde pendant plusieurs semaines. Mais du coup, celles-ci se retrouvèrent dans un espace qui était déjà, jusqu'à un certain point, un espace de conception. ${ }^{4}$

13 Les deux journaux considérés de Cocteau et de Bailly ont donc été composés avec la perspective probable d'une publication, et il suffit pour parachever leur transformation en livre d'ajouter un paratexte. Ils reçoivent un titre: Phèdre en Inde fait référence synthétiquement à l'expérience théâtrale qui a suscité le voyage et le journal ; Maalesh fait référence à une expression arabe très courante en Égypte (maalesh signifiant «ce n'est rien, ce n'est pas grave, tant pis ») et dépayse d'emblée le lecteur. Maalesh était également le titre d'une revue francophone égyptienne, «Journal politique, humoristique paraissant le samedi », dont Cocteau a pu avoir connaissance. Autres éléments du paratexte: une dédicace chez Cocteau «A S. E. Mohamed Wahid-el-Din » et une note liminaire explicative chez Bailly.

14 Écrits à quarante ans de distance, ces journaux de voyage témoignent d'un regard différent porté sur le monde. Maalesh est le journal d'un homme de lettres connu, qui porte encore l'empreinte de l'époque coloniale. Cocteau ignore la modélisation (fréquente, en revanche, chez Bailly) et ose des généralisations naïves que plusieurs décennies de décolonisation rendent malsonnante :

L'Égyptien est très susceptible. (M, p. 49)

Ce peuple qui flâne et qui dort dans la poussière, adopte, par mimétisme, les teintes inimitables du sable, du ciel, de l'eau du Nil. Faite pour la paresse et la mort, plus longue et plus sûre que la vie, cette race que le café, le hachich et le thé noir énervent, se partage entre une activité de cour de collège et la petite mort du sommeil. (M, p. 35)

Cocteau est mû par un projet qui consiste à exporter en Orient un panorama de la culture théâtrale occidentale, de Racine à Sartre en passant par Feydeau, Anouilh et lui-même. Accompagné de sa troupe, sa «roulotte céleste» $(M$, p. 25$)$, sa «famille d'acteurs» ( $M$, p. 25), Cocteau transporte avec lui son environnement privilégié. Les pièces, interprétées par des comédiens français, sont jouées devant un public que le diariste évoque sommairement, pour évaluer la réception qui est faite des différentes pièces. L'on peut d'ailleurs supposer que le public de 1949, des salles d'Alexandrie, Beyrouth et Istanbul, est pour sa majorité constitué d'occidentaux expatriés et d'une minorité de privilégiés de la population locale. Lors de son séjour en Égypte, Cocteau a essentiellement côtoyé le milieu aristocratique égyptien, naturellement francophone ${ }^{5}$. C'est de façon manichéenne que Cocteau décrit la population égyptienne : 
D'un côté, une société s'accroche à l'Égypte mondaine, l'Égypte du Sporting, du tennis et du golf. De l'autre, un peuple fanatique dissimule, sous la course au pourboire, sa haine xénophobe. Entre les deux, les nouveaux riches roulent carrosse et remplissent notre théâtre. Une élite aime la France et s'affecte de voir sa propagande mal faite (nulle). ( $M$, p. 88)

L'emploi même du terme de "propagande » ne manque pas de déranger un lecteur du $\mathrm{XXI}^{\mathrm{e}}$ siècle mais ne saurait finalement surprendre sous la plume d'un artiste dont les positions politiques et idéologiques sont sans doute représentatives d'une certaine bourgeoisie conservatrice des années 1940, encore attachée à ce que pouvait être le rayonnement colonial de la France. Cocteau revendique en particulier une meilleure diffusion des films français dans le Bassin méditerranéen et en déplore, à plusieurs reprises, les mauvaises conditions :

Il faudrait que la France se rendît compte de ce qu'elle représente et de ce qu'on attend d'elle. Mais elle ignore avec quel héroïsme un Bonneau enseigne notre langue à des paysans anatoliens qui viennent des montagnes désertes. Elle ignore que les Turcs, qui la parlent, la parlent mieux que nous-mêmes. Elle ignore que nos films, envoyés par la valise diplomatique, sauveraient notre prestige et seraient projetés dans tous les centres nerveux d'Ankara et d'Istanbul et que, grâce à ce système, le marché mort ressusciterait partout. ( $M$, p. 192)

17 Bref, quand bien même Maalesh est dédié au prince Mohamed Wahid-el-Din et quand bien même cet Égyptien est présent dans le journal ainsi que quelques autres, les contacts établis par Cocteau avec la population des pays traversés semblent pour le moins superficiels, souvent réduits au jeu des mondanités. La publication de Maalesh provoqua un fort mécontentement en Égypte, où l'ouvrage fut frappé d'interdiction de diffusion. Une entrée telle que celle du 4 avril ne pouvait que susciter l'irritation du pouvoir: «Cette nuit au désert, sous la tente de la princesse F. nous avons trop bu. L'hôtel ne nous réveille pas. Bousculade. C'est le style de mon tour du monde » (M, p. 98). Maalesh suscita également les sarcasmes de certains intellectuels français tel Étiemble, fin connaisseur de l'Égypte où il a séjourné et travaillé de 1943 à 1948. Ce dernier a consacré à Maalesh un article à charge dans Les Temps modernes où il stigmatise, tour à tour, l'intérêt dérisoire d'un journal d'humeurs, l'ignorance de Cocteau qui le conduit à proférer lieux communs et absurdités sur l'Égypte pharaonique, son manque d'honnêteté intellectuelle, sa désinvolture choquante de privilégié, son absence du sens de l'observation, son manque de délicatesse vis-à-vis de ses hôtes. Cocteau serait dans l'optique d'Étiemble la caricature même du voyageur .

18 C'est une toute autre image de diariste que construit Bailly, quelque quatre décennies plus tard: celle d'un intellectuel contemporain qui prend soin de s'afficher comme un esprit de l'ère post-coloniale, qui souhaite susciter une véritable confrontation entre Orient et Occident et "étranger » Phèdre. La vulgarisation de l'ethnographie, comme ouverture à l'altérité, dans la culture commune transparaît dans les journaux de voyage de diaristes contemporains qui ne sont pas à proprement parler des ethnographes... et il est évident qu'un Jean-Christophe Bailly, s'il devait invoquer des prédécesseurs, se réclamerait plus du Leiris de L'Afrique fantôme que du Cocteau de Maalesh.

19 Ces deux manières d'être suscitent des écritures diaristiques différentes. Les deux diaristes sont certes saisis par «la violence pure de l'impression » qui est aussi celle du dépaysement radical, mais leur regard ne s'arrête pas sur les mêmes réalités. Chez Cocteau, le regard est volontiers panoramique: «Égypte! Je vois une chose plate et de profil, étincelante, comme les marais salins d'Alexandrie » ( $M$, p. 81) ou encore : 
Dans les environs de Beyrouth, dans ce faste largement aéré qui commence par des pelouses de verdure, de sable orange et de pins parasols, jusqu'aux neiges et Baalbek dont les colonnes pendent du ciel, en passant par le méandre des routes parentes de nos routes des Alpes, on s'étonne d'une similitude avec nos campagnes françaises. (M, p. 139)

Quand il n'est pas panoramique, le regard coctalien est retenu par le monumental : les pyramides de Snefron ( $M, p .92)$, les Sphinx, « haie de béliers dont le visage vicieux et grave rappelle celui d'Aménophis IV » (105), le temple de Karnak, les colosses de Memnon ( $M$, p. 108), le temple de Louxor ( $M$, p. 114). À la fin du séjour égyptien, Cocteau conclut : "J'emporte d'Égypte la reconnaissance pour une hospitalité permanente et une mélancolie de n'être pas un égyptologue, c'est-à-dire totalement abstrait des problèmes insolubles qui embrouillent le monde moderne ( $M$, p. 132). L'Égypte, contemporaine, musulmane et arabe, ne l'intéresse guère.

Chez Bailly, l'Inde est saisie de manière kaléidoscopique... Le diariste écrit à une époque post-perecquienne où l'infra-ordinaire a conquis ses lettres de noblesse et où l'anodin mérite d'être noté. Se méfiant des "appréciations d'ordre général », porteuses « d'une impression de fausseté, de tromperie » (PhI, p. 57), Bailly accumule les observations, notées en désordre, sans tenter aucune interprétation prématurée : "Même si rien ne s'échappe du kaléidoscope secoué qu'une série d'images montées à la hâte et peut-être de travers, l'on se dit que c'est par ce chemin que l'on est passé et qu'il faudra encore en passer encore par lui avant de pouvoir avancer un jugement» (PhI, p. 57). Alors que Cocteau ne fait que passer dans des lieux glorieux, Bailly séjourne à trois reprises à Bhopal, vaste ville du centre de l'Inde, d'abord connue par l'immense catastrophe industrielle $e^{7}$ qui l'a endeuillée en 1984. C'est là qu'il doit diriger la mise en scène de Phèdre par une troupe indienne. Les séjours successifs dans une ville qu'on pourrait qualifier d'atouristique permettent un véritable processus d'apprivoisement qui passe par la visite rituelle du vieux marché et du New Market :

Dans la ville nouvelle, les vaches s'abritent parmi les hommes sous les galeries couvertes qui longent une avenue défoncée, immobiles et magiques, si étrangement chez elles devant les étals, avec leurs yeux de bonnes et douces bayadères dénuées de convoitise. Au marché, les fruits et les légumes, en pyramides, brillent sous la pluie, comme des trésors. Réseau des petites lampes fragilement sur les flaques. ( $P h I$ , p. 28, 6 août)

Promenade en rickshaw puis à pied dans le Chowk, le bazar, le cœur de la ville, sous une pluie tantôt violente, tantôt éclaircie. (PhI, p. 28, 7 août)

Traîné le matin au New Market, presque familier désormais. (PhI, p. 43, 12 août)

Tour au New Market. Des enfants vendent des tuyaux de plastique perforés qui, remplis de poudres colorées et roulés sur le sol, forment des frises régulières. (PhI, p. 82,7 novembre)

Après la séance du soir, dîner au New Market avec trois acteurs, dans le restaurant aux petites lampes vertes où les quelques bouteilles d'alcool entreposées derrière un comptoir suffisent à donner au lieu un air de contrebande. (PhI, p. 96, 13 novembre)

Vieux marché (Chowk). Vaches couchées devant les orfèvres assis. Près de Jasma Masjid, on tâte la jambe d'une petite fille, qui a été heurtée par un scooter, elle pleure au milieu d'un petit attroupement, deux gros Sikhs klaxonnent dans leur Maruti, avec cet inimitable air de brutes qu'ils ont parfois (tels ceux qui rossent Tintin dans le Lotus bleu). Chaleur douce du soleil d'hiver, la rue des verriers est pleine de paille, passe une chèvre aux cornes peintes en bleu, tout semble détendu, revenu à une demi-léthargie. Le seul défaut du bazar de Bhopal, c'est qu'il n'est pas assez grand pour qu'on puisse s'y perdre. (PhI, p. 119, 13 décembre) 
New Market. Achat de bananes, d'une guirlande de fleurs, de petites lampes à huile. Dans le contact raréfié avec l'extérieur auquel le travail nous contraint, ces brèves incursions matinales sont plus que des bouffées d'air: des parenthèses magiquement ouvertes sur un là-bas qui serait soudain ici-même. (PhI, p. 125, 16 décembre)

Jour de repos pour les acteurs. Pluie et brume sur les chantiers et les arbres toujours verts. Promenade au bazar et dans la vieille ville. (PhI, p. 137, 25 décembre) Dernière promenade dans Bhopal, en partant du Vieux Marché, derrière Hamydia Road, où des buffles piétinent dans un mélange de boue et de feuilles de salade. Le soleil, très vif, hausse le ton des couleurs, et le bazar, par les rues qui remontent vers Jama Masjid, me semble plus gai et plus vivant que de coutume. Je suis ému, tout simplement parce que je pars sans savoir si je reviendrai jamais, quoiqu'une dette soit contractée ici pour moi, envers cette ville rétive et sans doute malade, mais qui aura pourtant été notre « porte de l'Inde ». (PhI, p. 156-157, 6 janvier)

Certes les journaux de voyageur en disent un peu sur les lieux traversés; ils en disent sans doute encore plus sur les diaristes et l'image qu'ils donnent d'eux-mêmes à travers leurs lignes. Cocteau dont on a tant exhaussé la figure de poète virtuose très sollicité et d'acrobate trop doué, portrait qui l'insupportait, a écrit nombre de pages dans ses journaux personnels pour réparer l'image de touche-à-tout dilettante qui lui collait à la peau. Il y est parvenu parfois comme dans le Journal qui accompagne le tournage de $L a$ Belle et la Bête. De ce point de vue, Maalesh abîme l'image plus qu'il ne la répare, et si l'on ne peut pas reprocher à Cocteau un autoportrait apprêté, l'on peut simplement noter la naïveté de l'Occidental, dépourvu de mauvaises intentions mais infatué de la conscience de sa supériorité. Cela dit, on peut avancer que le journal de voyage, au-delà d'une idiosyncrasie et d'une individualité, nous éclaire aussi sur le rapport à l'autre et à l'étranger que chaque culture et chaque époque suscitent. Cocteau est sans doute assez représentatif de la mentalité de maints de ses contemporains. Rien de naïf, en revanche, dans les journaux de voyage intellectualisés de Bailly. Si l'on ne peut que saluer la subtilité de certaines analyses et l'acuité de maintes évocations et si, comme il l'assure, la règle du jeu des journaux de voyage publiés est de les livrer à l'impression sans retouche ni corrections ${ }^{8}$, l'on se surprend néanmoins à penser que Bailly a intériorisé une manière de charte du journal de voyage : il partage les attentes d'un lectorat peu désireux d'être pris en flagrant délit d'occidentalocentrisme et y répond. La quatrième de couverture de son dernier journal de voyage publié 9 , qui s'appliquerait aussi bien à Phèdre en Inde, pourrait être le texte de cette charte du diariste qui se sait et se veut éclairé, délesté de tout préjugé et ouvert à la réalité observée, charte presque trop belle pour être vraie : 
Des impressions, des sensations, des idées en cheminement, en connexions tendues, leurs terminaisons nerveuses pour faire toucher du doigt l'espace physique de leur parution. Dans ces carnets de voyage, Jean-Christophe Bailly [...] nous délivre quelque chose de la grande extériorité du monde sud-américain, mais selon un usage de la vision qui s'élime par avance de tout exotisme et dégage la langue de l'emprise topique du reportage. Cette netteté du regard [...] manifeste une acuité au réel qui ne décolle pas l'apparence de la profondeur.

\section{NOTES}

1. J.-Ch. Bailly, Phèdre en Inde, Paris, Plon, « Carnets », 1990, p. 15 (dorénavant PhI). Les citations ultérieures de ce journal seront suivies, dans le texte même, de la référence à la page entre parenthèses.

2. J. Cocteau, Maalesh, Journal d'une tournée de théâtre, Paris, Gallimard, 1949, p.143-144 (dorénavant $M$ ). Les citations ultérieures de ce journal seront suivies, dans le texte même, de la référence à la page entre parenthèses.

3. J.-Ch. Bailly, Dans l'étendu, Lyon, Fage éditions, 2010, p. 5.

4. Ibid., p. 7.

5. Voir A. Youssef, Cocteau l'Egyptien. La tentation orientale de Jean Cocteau, Monaco, éd. du Rocher, 2001.

6. Voir extraits d'Étiemble, Chronique littéraire, «La, mouche mâlèche », Les Temps modernes, n. 54, avril 1950 :

«En vain chercheriez-vous, dans Maalesh, qui se prétend le journal d'une tournée, la plus discrète allusion à l'article de Bishr Farès sur Les Parents terribles (il était pourtant assez dur, et mémorable). N'espérez donc pas y lire quelque pressentiment de l'opinion de Jean Cocteau de ceux qui dans les lettres françaises ont quelque crédit en Égypte. Avec ses airs de somnambule, l'auteur est extra-lucide. Il ne dit que ce qu'il veut dire [...].

Passons sur l'indécence du battage commercial : désormais, chaque fois que Cocteau monte une pièce ou tourne un film, nous n'y coupons pas de ses futiles aveux : au journal d'un film, qui nous renseignait sur les furoncles de Jean Marais, voici succéder Maalesh qui nous renseigne sur le génie de Jean Marais, et sur quelques dîners en ville, ou au désert [...].

Il est à craindre que Maalesh ne fasse à Cocteau plus de tort qu'à l'Égypte. On se gaussera des faiblesses de ce journal: de tant d'ignorance avec soin étalée. Car il a des idées sur tout, le voyageur [...].

Et gogo, avec ça. Aveugle à cette vie de la nécropole thébaine, il n'a d'yeux que pour les schémas d'une équipe d'occultistes qui entend répéter - à propos des temples de Louqsor et de Karnak les bobards de l'Abbé Moreux sur les "secrets" de la Grande Pyramide. Avant de me livrer aux sortilèges des Lubicz, j'aurais pris la précaution de lire au moins l'essai de Maspéro sur le temple de Louqsor, le livre de Lauer sur les pyramides et les temples de Saqarah. [...] Gogo, mais imposteur aussi. [...] Dans ce même temple de Louqsor décidément fertile en miracles et dont la baronne de Lubicz venait de lui révéler le vrai plan (un "squelette adulte"), "brusquement" à la salle hypostyle, Cocteau fait halte. [...]" En l'air, sur la haute muraille, Rimbaud a gravé son nom etc. ". Voyez-vous ça ! Quel flair, ce Cocteau ! Quel découvreur ! [...].

Entre deux coquetèles, à travers les vitres de sa voiture, ou de son avion, il a cru entrevoir l'autre bout de “l'échelle”. “ D’un côté, une société s'accroche à l'Égypte mondaine, l'Égypte du Sporting, 
$\mathrm{du}$ tennis et du golf. De l'autre, un peuple fanatique dissimule, sous la course au pourboire, sa haine xénophobe. Entre les deux, les nouveaux riches roulent carrosses et remplissent notre théâtre. Une élite aime la France et s'affecte de voir sa propagande mal faite (nulle)". Mais il connaît moins bien qu'il ne fait les oisifs du Caire ce peuple à l'en croire "fanatique" et "xénophobe" [...]. Qu'il veuille donc bien considérer que beaucoup d'étrangers qui vivent en Égypte sont toujours colonialistes. Que, secouant aujourd'hui tant d'humiliations, certains Égyptiens se portent à l'excès, nous pouvons en souffrir, mais il faudrait comprendre. Comprendre, aussi, que la "xénophobie" chez tout peuple qui fut colonisé, c'est le premier réveil d'une révolte qui, mieux orientée, peut devenir féconde [...].

À vrai dire, Jean Cocteau, qui coquette aujourd'hui avec Louis Aragon et Les Lettres françaises, me paraît dandysto-marxiste : "Aucun costume de théâtre" n'égale pour lui en beauté les haillons de la misère cairote. "Cette détresse, dit-il encore, n'en est pas une comme elle le serait en France", parce qu'on y trouve toujours quelque chose de "facile, de détendu... quelque chose, en quelque sorte de fastueux". Ça vous a un petit air extrêmement intelligent. Ça ne l'est pas [...].

Maalesh ! Cocteau m'excusera, si je réponds : La, mouche mâlèche (note : Laa, mies ma'alesh, dit l'Égyptien; l'Européen, en son arabe de petit-blanc: La, mouche mâlèche). Non, ça ne fait pas rien. J'espère néanmoins que, le prenant au mot, les Égyptiens en souriront: "Maalesh? ... maalesh!"».

7. C'est dans l'usine de pesticides d'Union Carbide que s'est produite en 1984 l'une des plus graves catastrophes industrielles connues (plus de 20.000 morts victimes des gaz toxiques).

8. Voir J.-Ch. Bailly, Dans l'étendu, cit., p. 8.

9. Ibid., quatrième de couverture.

\section{INDEX}

Mots-clés : Cocteau (Jean), Bailly (Jean-Christophe), Maalesh, Phèdre en Inde, journal de voyage 